\title{
Vulnerabilidade da rede viária urbana: avaliação considerando risco e emissão de gases de efeito estufa
}

\author{
Urban road network vulnerability: assessment considering risk and greenhouse \\ gas emissions
}

George Vasconcelos Goes ${ }^{[0]}$, Márcio de Almeida D’Agosto ${ }^{[0]}$, Bruno Vieira Bertoncini[ ${ }^{[b]}$, Gérsica Vasconcelos Goes ${ }^{[]}$

\footnotetext{
[a] Universidade Federal do Rio de Janeiro (UFRJ), Instituto Alberto Luiz Coimbra de Pós-Graduação e Pesquisa de Engenharia (COPPE), Programa de Engenharia de Transportes (PET), Rio de Janeiro, RJ, Brasil

[b] Universidade Federal do Ceará (UFC), Programa de Pós-graduação em Engenharia de Transportes (PETRAN), Fortaleza, CE, Brasil

[c] Universidade de Fortaleza (UNIFOR), Centro de Ciências Tecnológicas, Fortaleza, CE, Brasil
}

\section{Resumo}

Uma rede é vulnerável quanto maior for a externalidade negativa associada à ineficiência de um ou mais arcos. Dessa forma, existem, na literatura, métodos que podem ser utilizados para a análise de vulnerabilidade de uma rede viária, sendo divididos em dois grupos: de cálculos completos e com critérios predefinidos. Os de cálculos completos fazem uma varredura na rede em busca dos arcos mais vulneráveis, enquanto aqueles com critérios predefinidos utilizam procedimentos para obtenção desses arcos. Este artigo apresenta um procedimento que busca verificar a efetividade do risco de acidente como um critério predefinido para localizar os arcos mais vulneráveis de uma rede viária, avaliando o aumento da distância e da emissão total de $\mathrm{CO}_{2}$-eq em relação ao cenário base. Os resultados mostraram um aumento de até 8,51\% da distância de viagem quando são rompidos arcos com maior risco de acidentes. $0 \mathrm{CO}_{2}$-eq seguiu a mesma tendência, ou seja, rupturas em arcos com alto risco causaram um aumento de até $9,19 \%$, enquanto o aumento das emissões após o rompimento de arcos com baixo risco foi de apenas 5,54\%. Portanto, a quantidade de cenários modelados para coleta de dados e a simulação de Monte Carlo foram eficazes em avaliar os impactos ao longo do tempo.

Palavras-chave: Engenharia de tráfego. Vulnerabilidade. Risco. Áreas urbanas. Gases de efeito estufa.

\section{Abstract}

A network is vulnerable when the inefficiency of one or more links exerts negative externalities that may compromise the accessibility. The literature presents procedures that can be used in the network vulnerability assessment, which are divided into two groups: complete calculation and pre-defined criteria. The first ones, scan

GVG é doutorando em Engenharia de Transportes (COPPE-UFRJ), pesquisador associado do Programa de Engenharia de Transportes da COPPE (UFRJ), e-mail: ggoes@pet.coppe.ufrj.br

MAD é doutor em Engenharia de Transportes pela COPPE (UFRJ), professor do Programa de Engenharia de Transportes da COPPE (UFRJ), e-mail: dagosto@pet.coppe.ufrj.br

BVB é doutor em Engenharia de Transportes, pela Escola de Engenharia de São Carlos (USP), professor do Departamento de Engenharia de Transportes da Universidade Federal do Ceará, e-mail: bruviber@det.ufc.br

GVG é doutoranda em Arquitetura e Urbanismo (UFC), professora da Universidade de Fortaleza, e-mail: gersica@unifor.br 
the network in order to find the most vulnerable links, on the other hand procedures that consider predefined criteria use indirect approaches to obtain the vulnerability. This paper presents a framework to road network vulnerability analysis based on the risk and environmental aspects. Hence, we developed a new procedure to verify the effectiveness of risk as an attribute to find the most vulnerable links of a road network, considering distance and $\mathrm{CO}_{2}$-eq emissions. Results showed an increase up to $8.51 \%$ (total distance traveled) when links with high risk are disrupted. $\mathrm{CO}_{2}$-eq emissions yielded the same outcome, disruptions of high risk links caused an increase up to $9.19 \%$, whereas the safest links caused an average increase of only 5.54\% when disrupted. Therefore, the number of scenarios modeled (for collecting data) as well as the Monte Carlo simulation were effective in assessing the impacts over time.

Keywords: Traffic engineering. Vulnerability. Risk. Urban áreas. Greenhouse gas.

\section{Introdução}

O avanço contínuo do processo de urbanização em todo o mundo é responsável pelo aumento da atividade de transporte e da intensidade do uso de veículos nos centros urbanos. Esse fenômeno tem contribuído para a saturação da relação entre o fluxo de veículos e a capacidade dos sistemas de transporte, aumentando a frequência e a duração dos congestionamentos em áreas densamente povoadas. Esse cenário é potencializado em vista de os sistemas de transporte serem configurados para operarem próximos à capacidade como forma de reduzir os custos (Mattsson \& Jenelius, 2015). Assim, o menor distúrbio na rede viária pode gerar impactos econômicos, sociais ou ambientais que, inicialmente, são percebidos pelos usuários por meio dos congestionamentos de tráfego.

Apesar das políticas públicas quanto à ocupação do solo e à mobilidade urbana, observa-se que as externalidades negativas, em especial a intensificação do transporte motorizado individual, têm gerado incrementos sucessivos do volume de tráfego no sistema de transporte. Posto isso, o crescimento da demanda por viagens em áreas urbanas impõe pressões para a expansão constante da capacidade do sistema (Bevrani et al., 2017), o que causa grandes implicações em termos do uso da energia e de emissões de gases de efeito estufa (GEE) e poluentes na atmosfera (Lyons, 2016).

A literatura define dois tipos de congestionamento de tráfego: (1) recorrente, se é dependente dos fluxos e da densidade de tráfego; e (2) não recorrente, quando é resultado de eventos externos, como um acidente (Conca et al., 2016). Dessa forma, a importância de um sistema de transportes robusto e confiável gera o desenvolvimento de pesquisas com objetivo de compreender os mecanismos e as inter-relações que criam a sua vulnerabilidade, bem como formas de torná-lo resiliente para mitigar as consequências de perturbações (Mattsson \& Jenelius, 2015).

Ao considerar que a medição da vulnerabilidade passa pela redução ou obstrução da capacidade dos arcos e pela análise do impacto em termos de aumento do tempo da viagem ou dos custos monetários (Jenelius \& Mattsson, 2012), existem, na literatura, métodos que podem ser utilizados e que são divididos em dois grupos (Knoop et al., 2012). 0 primeiro grupo contém os métodos de cálculos completos, ou seja, a capacidade é reduzida para cada arco individualmente. Dessa forma, faz-se uma varredura na rede viária para descobrir quais arcos são mais críticos em caso de interrupções. 0 segundo grupo utiliza critérios predefinidos para ter uma direção na busca de elos mais vulneráveis. Nessa abordagem, primeiro se selecionam arcos que são possivelmente vulneráveis, baseados em características específicas (por exemplo, uso do solo, topologia, condições de acessibilidade, histórico de desastres etc.). Para esses arcos, uma análise mais detalhada é realizada por meio da redução da capacidade e, com isso, avalia-se a vulnerabilidade de cada arco. Desenvolver métodos que se enquadrem no segundo grupo é interessante do ponto de vista da modelagem de rede, no intuito de reduzir iterações no processo de simulação computacional do experimento.

Pode-se, portanto, identificar os arcos críticos da rede viária de forma indireta, ou seja, que não necessite reduzir a capacidade individualmente de todos os arcos de uma rede. Esses critérios adicionais motivam o desenvolvimento de procedimentos para a determinação de indicadores auxiliares, com 
validade para toda a rede, independente do grau de complexidade do sistema (Oliveira et al., 2016).

Dessa forma, o objetivo deste estudo é verificar a eficácia de um atributo de risco de acidente como direcionador para a análise de vulnerabilidade da rede viária, em termos de aumento da distância e da emissão total de $\mathrm{CO}_{2}$ equivalente ( $\mathrm{CO}_{2}$-eq), com ênfase no transporte urbano de carga. Para isso, realizou-se uma Revisão Bibliográfica Sistemática (RBS) com o propósito de identificar, na literatura especializada, quais fenômenos de interrupção são utilizados em análises de vulnerabilidade, o cenário (urbano ou regional), as métricas, os métodos e os aspectos ambientais (mitigação de GEE). É importante salientar que a versão com resultados preliminares do estudo foi apresentada nos anais do XXXI Congresso Nacional de Pesquisa e Ensino em Transporte da ANPET, de 2017 (Goes et al., 2017).

0 trabalho foi dividido em seis seções, além desta primeira: a seção 2 discute o conceito de risco na análise de vulnerabilidade; a seção 3 mostra o resultado da revisão bibliográfica sistemática sobre a análise de vulnerabilidade de uma rede viária; a seção 4 aborda o método desenvolvido nesta pesquisa para análise de vulnerabilidade da rede viária; na seção 5 , apresentam-se a aplicação do procedimento e os resultados encontrados; por fim, a seção 6 discute as conclusões do trabalho e as recomendações para trabalhos futuros.

\section{Risco na análise de vulnerabilidade}

Risco é um conceito utilizado para caracterizar a ameaça de um evento de desastre em termos de probabilidade de ocorrência e das consequências (Faturechi \& Miller-Hooks, 2014). 0 método deste trabalho para mensurar o valor esperado do risco é baseado no conceito exposto por Haimes (1998), o qual é ainda pertinente a problemáticas contemporâneas. O autor afirma que o valor esperado do risco é essencialmente o produto das consequências de cada evento pela probabilidade de ocorrência e, ao final, somam-se todos os produtos no universo de eventos possíveis. Dessa forma, para cada cenário de risco concebível, a avaliação pode ser formalizada como um "tripleto": (1) descrição de cenário; (2) probabilidade; e (3) consequências (medida de dano) desse cenário (Mattsson \& Jenelius, 2015). As consequências dizem respeito a impactos socioambientais e econômicos. No tocante à probabilidade e exposição ao risco de acidentes (objeto deste trabalho), foram desenvolvidos os Modelos de Previsão de Acidentes (MPA), que, entre outros objetivos, buscam aferir o risco de acidentes para determinado trecho, ou interseção, mediante variáveis que expliquem o grau de incidência ao risco (Cardoso \& Goldner, 2007).

0 atributo de vulnerabilidade, por sua vez, busca inferir o impacto no desempenho de uma rede viária após a obstrução total ou parcial de um arco ou nó. Dessa forma, considera as potenciais consequências de um evento de desastre sobre o desempenho do sistema (Faturechi \& Miller-Hooks, 2014). A vulnerabilidade da rede de transporte pode ser vista como um problema de acessibilidade reduzida devido a diferentes perturbações nos arcos (Chen et al., 2007). Para Jenelius et al. (2006), o custo pode vir sob a forma de outras variáveis, como: distância, valor monetário etc.; contudo, evidencia-se a utilização do tempo de viagem como variável mais difundida nos modelos de vulnerabilidade. Nesse contexto, a literatura sugere que alterações no tempo de viagem de cada arco representam variações proporcionais do valor do custo generalizado da rede de transportes (Altiparmak et al., 2006; Gen et al., 2006; Taylor et al., 2006; Sullivan et al., 2010; Cheshmehgaz et al., 2013). Na visão de Yang et al. (2000), medidas baseadas em variabilidade de tempos de viagem (por exemplo, atributo de confiabilidade) são úteis para avaliar o desempenho da rede de transportes em termos de qualidade do serviço prestado aos usuários no dia a dia.

\section{RBS para a análise de vulnerabilidade da rede viária}

Para o desenvolvimento da RBS, seguiu-se a metodologia proposta por Thomé et al. (2016). A etapa 1 envolveu a seleção da base de dados. Optou-se pela Science Direct por ser um catálogo de periódicos indexados e revisados por pares. A etapa 2 consistiu na identificação de palavras-chave para pesquisa, resultando na seguinte estrutura: "vulnerability" E "transportation" E ("exposure" OU "risk"). A busca pelos termos foi aplicada no título, no resumo e nas palavras-chave dos artigos, com a restrição do período de 2007 a 2017. Restringiu-se também a artigos publicados em inglês em revistas indexadas 
e revisadas por pares, conforme recomendado por Nord \& Nord (1995) e Ngai \& Wat (2002), para garantir a qualidade dos estudos. Como resultado, foram identificados 105 artigos.

Na etapa 3, foram especificados os critérios de exclusão: artigos cujo conteúdo estava relacionado à análise de vulnerabilidade, mas não especificamente à acessibilidade (transporte). De acordo com esses critérios de exclusão, os resumos foram revisados por pelo menos dois pesquisadores (etapa 4), o que resultou na seleção de 25 trabalhos para revisão do texto completo. Após a revisão completa dos artigos (etapa 5), o número de trabalhos foi reduzido para 10. Os trabalhos foram, por fim, avaliados de acordo com os seguintes critérios:

- Fenômeno de interrupção: fator responsável pelo corte no arco;

- Cenário: transporte urbano ou regional;

- Procedimento para análise: cálculos completos (rupturas em todos os arcos da rede viária); grupo de arcos (rupturas de acordo com critérios predefinidos);

- Indicador: tempo de viagem; distância; valor monetário;

- Método: análise estatística (modelos paramétricos e não paramétricos, análise de regressão e modelos de risco); modelo probabilístico (modelagem dinâmica e redes bayesianas); teoria de redes complexas (por exemplo, teoria dos grafos); e simulação (por exemplo, Monte Carlo);

- Aspectos ambientais: estimação de emissões de GEE ou poluentes atmosféricos.

A Tabela 1 resume os resultados da RBS. Nota-se que nenhum dos trabalhos aborda a vulnerabilidade sob o âmbito das emissões de GGE ou poluentes, possivelmente em razão de os trabalhos com vulnerabilidade de uma rede viária explorarem os aspectos ambientais sob a ótica da adaptação (perante mudanças climáticas ou desastre recorrente), dando pouca ênfase à mitigação resultante de alterações de fluxos para caminhos mais distantes. Apenas dois trabalhos abordaram a utilização de critérios predefinidos para a escolha dos arcos a serem rompidos, dos quais apenas um considerou acidentes de tráfego como fenômeno de risco responsável pela ruptura do arco, mas que não considerou simulação no processo de análise, indicando uma contribuição inovadora desse artigo.

Com exceção do estudo de Ambituuni et al. (2015), todos os artigos levantados trataram o tempo de viagem como indicador principal para a análise de vulnerabilidade. Esse dado é esperado, pois, usualmente, o tempo de viagem é o principal fator de influência do custo da viagem a ser analisado em trabalhos de modelagem de tráfego (Leng et al., 2018), sendo um dos componentes do custo generalizado (Balijepalli \& Oppong, 2014). No entanto, ainda conforme

Tabela 1 - Resultados da RBS

\begin{tabular}{|c|c|c|c|c|c|c|}
\hline Autor & $\begin{array}{c}\begin{array}{c}\text { Fenômeno } \\
\text { de }\end{array} \\
\text { interrupção }\end{array}$ & Cenário & $\begin{array}{l}\text { Procedimento } \\
\text { para análise }\end{array}$ & Indicador & Método & $\begin{array}{l}\text { Aspectos } \\
\text { ambientais }\end{array}$ \\
\hline (Knoop et al., 2007) & Falhas técnicas & Regional & Cálculos completos & Tempo de viagem & Simulação & - \\
\hline (Luathep et al., 2011) & Congestionamentos & Urbano & Cálculos completos & Tempo de viagem & Redes complexas & - \\
\hline (Demirel et al., 2015) & Desastres naturais & Regional & Critérios predefinidos & Tempo de viagem & Simulação & - \\
\hline (Ambituuni et al., 2015) & Acidentes de tráfego & Regional & Critérios predefinidos & Valor monetário & Modelo probabilístico & - \\
\hline (Kermanshah \& Derrible, 2016) & Desastres naturais & Urbano & Cálculos completos & Tempo de viagem & Simulação & - \\
\hline (Inanloo et al., 2016) & Congestionamentos & Urbano & Cálculos completos & Tempo de viagem & Modelo probabilístico & - \\
\hline (Muriel-Villegas et al., 2016) & Desastres naturais & Urbano & Cálculos completos & Tempo de viagem & Modelo probabilístico & - \\
\hline (Asadabadi \& Miller-Hooks, 2017) & Desastres naturais & Urbano & Cálculos completos & Tempo de viagem & Modelo probabilistico & - \\
\hline (Thorisson \& Lambert, 2017) & Acidentes de tráfego & Urbano & Cálculos completos & Tempo de viagem & Análise estatística & - \\
\hline (Fialkoff et al., 2017) & Congestionamentos & Regional & Cálculos completos & Tempo de viagem & Análise estatística & - \\
\hline
\end{tabular}

Fonte: Elaborada pelos autores. 
Balijepalli \& Oppong (2014), abordagens para análise de vulnerabilidade baseadas na distância são bem empregadas em redes com poucas opções de escolha de rota (por exemplo, regionais). Apesar de não aparecerem na busca da RBS, é importante salientar os resultados de Cunha (2014) e Oliveira et al. (2016), que utilizaram critérios predefinidos para selecionar os arcos para interrupções no cenário nacional. 0 primeiro baseou-se em arcos críticos que pertenciam às principais rotas de escoamento de soja (via banco de dados) para o transporte regional de carga. Nesse estudo, o valor monetário foi o principal indicador de vulnerabilidade analisado. 0 segundo trabalho utilizou, em conjunto, um tripleto de indicadores de congestionamento, confiabilidade e quantidade de rotas entre centroides em meio urbano adensado, considerando o tempo de viagem e a confiabilidade como indicadores para a análise de vulnerabilidade da rede.

Nessa linha, o presente estudo considera o tempo de viagem para alocação dos veículos na rede viária, sendo fator de influência na decisão de qual rota escolher após uma ruptura (Atividade 1 do método do estudo). Ademais, para efeito da análise da vulnerabilidade, considerou-se somente o indicador de distância total (pós-ruptura), que representa o aumento da quilometragem total percorrida após a ocorrência de uma interrupção em um ou mais arcos da rede, uma vez que se pretende analisar, concomitantemente, as emissões de $\mathrm{CO}_{2}$-eq, que variam diretamente com a distância/o consumo (Brasil, 2013). 0 risco de acidente, do tipo abalroamento/colisão, foi escolhido por ser típico de conflito de tráfego, pois envolve pelo menos dois veículos em circulação (Cardoso \& Goldner, 2007), tendo como importantes fatores a velocidade e o volume de tráfego. Na RBS, o risco não foi diretamente abordado por nenhum artigo, com exceção de trabalhos que buscavam relacionar variáveis do fluxo de tráfego, mas que não os inseriam diretamente em um MPA.

Portanto, além de considerar o tempo (para alocação do fluxo de veículos), a distância (para análise de vulnerabilidade) e o risco de acidente (por meio de um MPA) como critério direcionador, este artigo ainda contribui ao inserir as emissões de GEE na atmosfera resultantes de alterações das rotas dos veículos após uma interrupção/redução da capacidade do arco. Desse modo, colabora metodologicamente com políticas mitigatórias de emissões.

\section{Procedimento}

O procedimento utilizado para análise de vulnerabilidade neste trabalho foi baseado no estudo de Goes et al. (2017). A Figura 1 ilustra as etapas

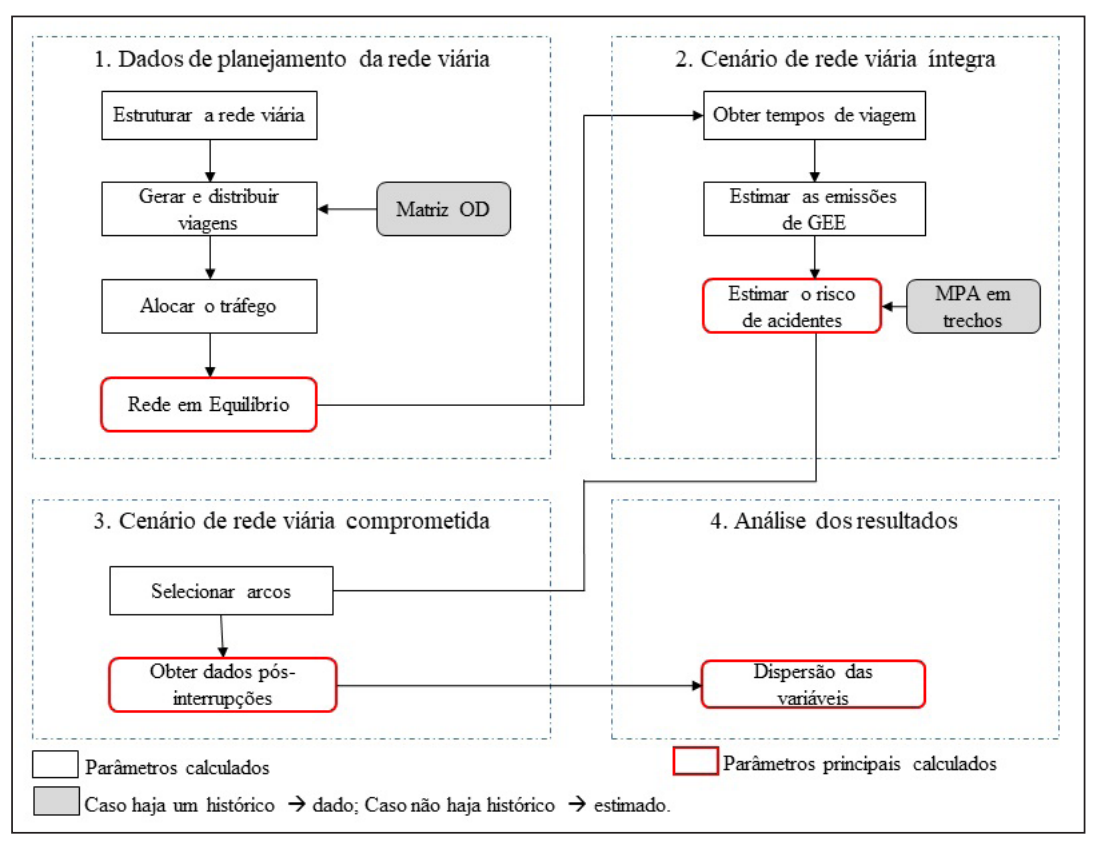

Figura 1 - Análise da vulnerabilidade por meio do direcionador de risco Fonte: Adaptada de Goes et al. (2017). 
metodológicas seguidas. 0 procedimento utilizado se aplica a redes viárias urbanas com presença de congestionamentos. As atividades consistem em:

- Dados de planejamento da rede viária - por meio das etapas 1.1, 1.2 e 1.3;

- Cenário de rede viária íntegra - etapas 2.1, 2.2 e 2.3

- Cenário de rede viária comprometida - etapas 3.1 e 3.2 ;

- Análise dos resultados - etapa 4.1.

\section{Aplicação do procedimento}

Este item busca expor as etapas metodológicas a serem seguidas para a análise de vulnerabilidade de uma rede viária, utilizando o risco como direcionador.

\section{Atividade 1: Dados de planejamento da rede viária}

0 procedimento foi aplicado em parte da rede viária da cidade de Fortaleza, no Ceará, Brasil, especificamente na região que abrange o bairro da Aldeota (Figura 2), área adensada que concentra grande quantidade de comércio e serviços, ou seja, há movimentação de carga urbana e presença constante de congestionamentos.

0 recorte da rede viária foi modelado no software de SIG voltado ao transporte TransCAD; contém 47 nós, conectados entre si por 67 arcos orientados. Os nós de origem (6,30 e 42) foram escolhidos por estarem próximos a zonas industrializadas. A escolha dos pontos de destino $(1,20$ e 47) se justifica por serem pontos estratégicos de recebimento de cargas. Os pontos 1 e 20 representam três shopping centers situados na área de abrangência. 0 ponto 47 representa a saída do sistema de transporte em direção à outra região comercial da cidade. A base georreferenciada (shape) foi cedida pelo Controle de Tráfego em Área de Fortaleza (CTAFOR), levando-se em conta dados de 2012.

Foram considerados 1.250 veículos por hora (vph) como capacidade para cada faixa de tráfego, conforme estudo realizado em vias urbanas semaforizadas apresentado por Colella et al. (2004). Nessa simulação, não se considerou a presença de interseções semaforizadas; embora seja importante, no tocante à microssimulação, a inserção desse fator, optou-se por simplificar essa etapa no estudo de caso.

O comprimento por arco foi padronizado em 113 metros, uma vez que representa a média dos comprimentos do recorte da região. Cada trecho possui entre quatro e cinco arcos, com velocidades máximas regulamentadas de $40 \mathrm{~km} / \mathrm{h}$ (coletoras) e $60 \mathrm{~km} / \mathrm{h}$ (arteriais). Desse modo, o tempo de viagem

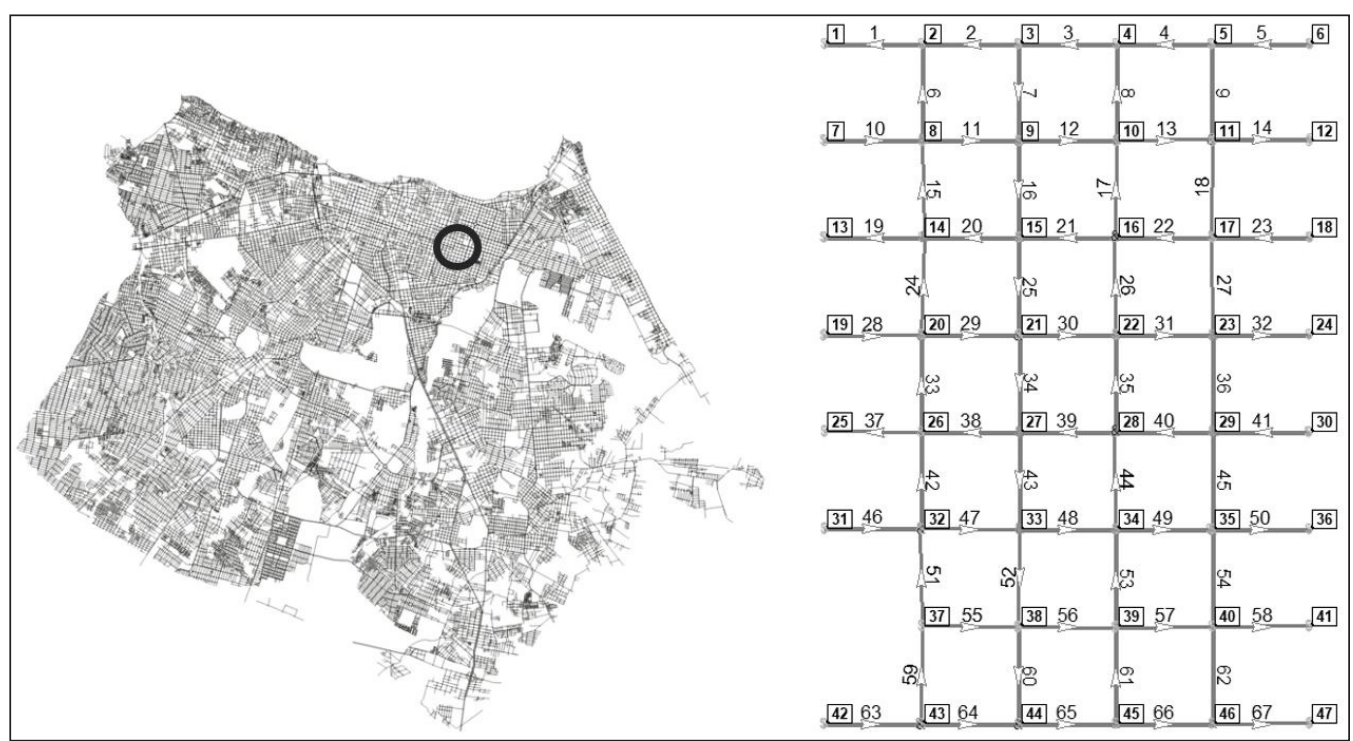

Figura 2 - Região do experimento e pontos OD distribuídos na rede Fonte: Goes et al. (2017). 
para cada arco em fluxo livre é de 6,78 segundos para as arteriais e 10,17 segundos para as coletoras. É evidente que, ao carregar a rede, o tempo de viagem, a quilometragem percorrida e a emissão de poluentes sofrerão variações, as quais podem influenciar na decisão da escolha da rota pelo usuário.

Em decorrência da limitação de acesso a dados e buscando não comprometer a confiabilidade da pesquisa, fez-se a avaliação de vulnerabilidade apenas com dados de emissão de veículos de carga, apesar de a alocação dos fluxos de veículos considerar transporte de passageiros e de carga. Entende-se que a etapa de alocação e de distribuição dos fluxos seja mais importante para a análise de vulnerabilidade de uma rede, tendo a mensuração das emissões oriunda de uma amostra de veículos validada como estudo científico. Assim, a movimentação dos veículos urbanos de carga (VUC) entre os pares origem-destino foi representada por matrizes OD de carga e de passageiros (Tabela 2).

Nesse experimento, a matriz OD de carga foi desenvolvida mediante a contagem de estabelecimentos comerciais e indústrias nos trechos da rede viária e teve seu volume inferido com base na quantidade de estabelecimentos comerciais e indústrias da região ( 2 shopping centers, 208 comércios e 2 indústrias). Dessa forma, foi obtida por um método expedito, desenvolvido internamente para realização do trabalho e sem qualquer garantia ou pretensão que ela representasse o real carregamento de VUC na área em estudo. Tratou-se de contagem in loco dos veículos de carga que viajaram entre os pontos OD no intervalo de 1 hora. Embora reconheça-se que uma matriz OD real traria maior contribuição ao método, entendeu-se que, devido ao objetivo ser o de verificar a aplicabilidade da proposta, os fluxos entre pares OD não seriam o elemento central de análise a ser considerado. Em complementação à matriz $0 \mathrm{D}$ de carga, utilizou-se a matriz OD de transporte de passageiros levantada no estudo de Goes \& Bertoncini (2016), com dados obtidos com o CTAFOR (dados de 2015) por meio de laços detectores nas interseções da região.

Para alocar o tráfego, adotou-se o modelo de equilíbrio determinístico do usuário, cujos preceitos iniciais foram desenvolvidos por Wardrop (1952). A decisão se baseou na utilização do modelo por outros autores da literatura especializada (Jenelius et al., 2006; Scott et al., 2006; Tampère et al., 2008; Sullivan et al., 2010; Huang et al., 2012); portanto, parte do princípio de que as realocações de usuários dispersos pela rede viária impactam no tempo de viagem dos arcos no sistema. Nesse tocante, a Função Objetivo $F$ busca minimizar o tempo total $t_{a}$ da rede de transportes, conforme as Equações 1 a 5.

$$
F=\sum_{a \in A} \int_{0}^{x a} t_{a}(y) d_{y}
$$

Sujeito à $\sum_{k=1}^{n i j} x_{i j}^{k}=T_{i j}^{k} \forall(i, j) \in \mathrm{OD}$

$x_{a}=\sum_{k=1}^{n i j} x_{i j}^{k} \sum_{(i, j) \in O D}\left(p_{i j}^{k}\right)_{a}\left(x_{i j}^{k}\right) \forall i \in \mathrm{A}$

$x_{i j}^{k} \geq 0 \forall k=\left(1, \ldots, n_{i j}\right)$

Em que:

$t_{a}$ : custo do arco $a$ (tempo);

$x_{a}$ : fluxo no $\operatorname{arco} a$;

$y$ : constante calibrada;

$T_{i j}$ : quantidade de usuários que desejam se locomover do nó $i$ para o nó $j$;

$x_{i j}^{k}$ : quantidade de usuários que percorrem $p_{i j}^{k}$ ( $k$-ésimo caminho entre $i$ e $j$ );

$p_{i j}^{k}=1$, se o arco $a \in p_{i j}^{k}$, ou 0 , caso não pertença.

0 custo do arco $t_{a}$ (medido em tempo) foi estimado conforme a Equação 5, proposta pelo Bureau of Public Roads (BPR), tratada na NCHRP 365 (TRB, 1998).

Tabela 2 - Matrizes de carga e passageiros

\begin{tabular}{cccccccccc}
\hline Origem/destino (nós) & $\mathbf{1}$ & $\mathbf{2 0}$ & $\mathbf{4 7}$ & $\boldsymbol{\Sigma}$ & Origem/destino (nós) & $\mathbf{1}$ & $\mathbf{2 0}$ & $\mathbf{4 7}$ & $\boldsymbol{\Sigma}$ \\
\hline 6 & 38 & 32 & 40 & $\mathbf{1 1 0}$ & 6 & 950 & 940 & 903 & $\mathbf{2 . 7 9 3}$ \\
30 & 54 & 48 & 44 & $\mathbf{1 4 6}$ & 30 & 750 & 650 & 670 & $\mathbf{2 . 0 7}$ \\
42 & 36 & 52 & 38 & $\mathbf{1 2 6}$ & 42 & 550 & 580 & 420 & $\mathbf{1 . 5 5}$ \\
$\Sigma$ & $\mathbf{1 2 8}$ & $\mathbf{1 3 2}$ & $\mathbf{1 2 2}$ & $\mathbf{3 8 2}$ & $\boldsymbol{\Sigma}$ & $\mathbf{2 . 2 5}$ & $\mathbf{2 . 1 7}$ & $\mathbf{1 . 9 9 3}$ & $\mathbf{6 . 4 1 3}$ \\
\hline
\end{tabular}

Fonte: Goes \& Bertoncini (2016). 
Mediante calibração prévia, foram adotados os valores 4 para a constante y e 0,15 para a constante $b$. 0 tempo inicial $t_{0, a}$ foi estimado com base na rede em condição de fluxo livre.

$t_{a}=t_{0, a}\left[1+b\left(\frac{f_{a}}{\text { cap }_{a}}\right)^{y}\right]$

Em que:

$t_{a}$ : tempo do $\operatorname{arco} a$;

$t_{0, a}:$ tempo inicial do arco $a$;

$f_{a}$ : fluxo no $\operatorname{arco} a$;

cap $_{a}$ : capacidade do arco $a$;

$y$ : constante de calibração;

$b$ : constante de calibração.

\section{Atividade 2: Cenário de rede viária íntegra}

Neste estudo, foram considerados os seguintes GEE: dióxido de carbono $\left(\mathrm{CO}_{2}\right)$, metano $\left(\mathrm{CH}_{4}\right)$ e óxido nitroso $\left(\mathrm{N}_{2} \mathrm{O}\right)$, uma vez que são os principais gases com potencial de aquecimento. Os fatores de emissão para $\mathrm{CO}_{2}(2,603 \mathrm{~kg} / \mathrm{l}), \mathrm{CH}_{4}(0,060 \mathrm{~g} / \mathrm{km})$ e $\mathrm{N}_{2} \mathrm{O}(0,030 \mathrm{~g} / \mathrm{km})$, para caminhão leve, foram obtidos no $2^{\text {}}$ Inventário Nacional de Emissões Atmosféricas por Veículos Automotores Rodoviários (Brasil, 2013). A eficiência energética $(4,99 \mathrm{~km} / \mathrm{l})$ foi obtida mediante coleta em campo (caminhão leve). Posteriormente, os dados de emissões foram convertidos em $\mathrm{CO}_{2}$-eq por meio da medida do potencial de aquecimento global (GWP) de cada gás, revisada periodicamente pelo Painel Intergovernamental sobre Mudanças Climáticas (IPCC) (Myhre et al., 2013).

A fim de estimar os arcos com maior risco de acidentes (como critério predefinido para direcionar a análise de vulnerabilidade), utilizou-se o MPA em trechos desenvolvido por Cardoso \& Goldner (2007), dado que representa um modelo de previsão de acidentes a partir de variáveis relacionadas à exposição e aos fatores de risco em um meio urbano adensado, cuja aplicação foi realizada em uma cidade de porte e com problemáticas de tráfego semelhantes (Porto Alegre, Brasil). Nesse modelo, utiliza-se uma distribuição de Poisson com função de ligação logarítmica, método utilizado por outros autores, como Lord et al. (2005). As variáveis significativas do MPA podem ser relacionadas à frota circulante (presença de motocicletas, caminhões pesados etc.), à via (qualidade do pavimento, comprimento, largura, interseções semaforizadas etc.) e ao fluxo de tráfego (velocidade média, volume etc.) que podem ser adotadas dependendo da disponibilidade de dados.

As variáveis significativas adotadas neste trabalho foram: volume médio diário de veículos (Vol_Veic) e velocidade média no trecho (Vel_Méd). A velocidade dos veículos no fluxo de tráfego é uma variável relevante em relação aos modelos de previsão de acidentes (Greibe, 2003; Aarts \& Van Schagen, 2006; Cardoso \& Goldner, 2007), tendo efeito tanto na probabilidade de ocorrência de acidentes quanto na severidade (TRB, 1998). Para Cardoso \& Goldner (2007), o volume de tráfego pode contribuir para a maior parcela de explicação na variabilidade de acidentes, podendo esclarecer a ocorrência de acidentes somente por meio da exposição ao risco, resultado também apontado no estudo de Greibe (2003). Assim, ambas as variáveis significativas são respaldadas pela literatura especializada como fatores de exposição relevantes; portanto, foram incorporadas ao estudo de caso. Após determinar as variáveis significativas, a próxima etapa foi baseada na codificação das variáveis para uma mesma base numérica, conforme Equação 6.

$\operatorname{Vcod}=\frac{\operatorname{Vobs}-A}{B-A}$

Em que:

$V_{c o d}$ : valor codificado da variável (intervalo de 0 a 1);

$V_{o b s}$ : valor observado para a variável;

$A$ : menor valor observado da amostra;

$B$ : maior valor observado da amostra.

Os fatores de risco (variáveis significativas) foram, por fim, inseridos na Equação 7.

$V R=e^{\left(b_{0}+\sum_{i=1}^{n} b_{x_{i}}\right)}$

Em que:

$V R$ : variável resposta;

$b_{o}$ : constante;

$b_{i}$ : parâmetros do modelo;

$x_{i}$ : variáveis significativas (fatores de risco e exposição analisados).

Adotaram-se os valores 1,4338 como o valor da constante $b_{0}, 0,7724$ para o parâmetro $b$ associado à variável Vol_Veic e -0,4388 para o parâmetro $b$ associado à variável Vel_Méd, os mesmos valores sugeridos por Cardoso \& Goldner (2007) em um centro urbano adensado. Tal formulação é necessária para atribuir uma gradação de risco (alto, médio e baixo) de cada 
arco da rede de transportes, considerando os fatores de risco (variáveis significativas) adotados.

Atividade 3: Cenário de rede viária comprometida

Após a aferição do risco de cada arco, tornou-se viável verificar se o atributo do risco pode direcionar a avaliação para os elos mais vulneráveis de uma rede viária. Foram simulados 32 cenários com o propósito de representar situações de falhas em arcos com índices extremos de risco de acidentes, identificando a variação na quilometragem percorrida e na emissão de $\mathrm{CO}_{2}$-eq em cada situação. A Tabela 3 mostra a variável resposta (VR) de cada arco do recorte da rede viária.

A análise da vulnerabilidade foi baseada na variação da quilometragem percorrida oriunda das mudanças de rotas em função das obstruções dos arcos. Isso posto, para efeito desta análise, quanto maior a variação da quilometragem percorrida na rede em relação ao cenário base de rede íntegra e quanto maior a emissão de $\mathrm{CO}_{2}$-eq, maior a vulnerabilidade que o arco representa à rede viária. Portanto, simulou-se a obstrução em 32 cenários: 16 com interrupções em arcos de alto risco e 16 em arcos de baixo risco.

A Tabela 4 evidencia os valores obtidos das médias amostrais de emissão e distância para que possa ser realizada uma simulação de Monte Carlo (Oliveira, 2001), visando obter aproximações numéricas de funções complexas. Considerou-se uma distribuição normal da amostra.

\section{Análise dos resultados}

Por meio da aplicação do procedimento desenvolvido neste trabalho, foi possível identificar o risco de acidente dos arcos de uma rede viária e verificar, pelo método Monte Carlo, se os valores extremos podem ser utilizados como direcionadores para análise da vulnerabilidade, considerando aspectos de distância total percorrida e de emissão total de $\mathrm{CO}_{2}$-eq (Figuras 3 e 4). Utilizou-se a ferramenta MS Excel para geração e tabulação dos números aleatórios (distribuição normal) com base nos parâmetros coletados na modelagem.

Na simulação, foram realizadas 1.000 iterações, utilizando os parâmetros de média amostral e desvio-padrão dos cenários de alto e baixo risco de acidente, ilustrados na Tabela 3. A Figura 3 mostra o confronto da quantidade emitida de $\mathrm{CO}_{2}$-eq pela distância total das viagens dos VUC da rede viária pós-incidente depois da simulação. Nota-se que as médias amostrais de emissão de $\mathrm{CO}_{2}$-eq e de distância total para o cenário de alto risco de acidente são mais elevadas do que no cenário de baixo risco.

Tabela 3 - Variável resposta por arco

\begin{tabular}{lcc}
\hline \multicolumn{1}{c}{ Arcos } & Variável resposta & \multicolumn{2}{c}{ Risco de acidentes } \\
\hline $9 ; 10 ; 11 ; 12 ; 13 ; 14 ; 15 ; 19 ; 20 ; 21 ; 22 ; 23 ; 24 ; 25 ; 29 ; 30 ; 32 ; 33 ; 34 ; 35 ; 39 ; 40 ; 49 ; 50 ; 57 ; 59 ; 60 ; 61 ; 62 ; 63 ; 64$. & VR $\leq 18$ & Baixo \\
$1 ; 2 ; 3 ; 5 ; 6 ; 7 ; 8 ; 16 ; 18 ; 26 ; 27 ; 28 ; 31 ; 36 ; 37 ; 38 ; 41 ; 46 ; 48 ; 53 ; 54 ; 56 ; 58 ; 67$. & $18<V R \leq 25$ & Médio \\
$5 ; 17 ; 42 ; 43 ; 44 ; 45 ; 47 ; 51 ; 52 ; 55 ; 65 ; 66$. & $25<V R$ & Alto \\
\hline
\end{tabular}

Fonte: Goes et al. (2017)

Tabela 4 - Parâmetros de entrada estocásticos para a simulação de Monte Carlo

\begin{tabular}{ccccc}
\hline Cenários & Emissão $\mathbf{C O}_{2}$-eq $(\mathbf{k g})$ & DP $^{3}$ & Distância percorrida (km) & DP \\
\hline Rede íntegra & $1.192,81$ & - & $2.285,25$ & - \\
Cenário |' & $\mu=1.291,70$ & $\sigma=71,10$ & $\mu=2.474,71$ & $\sigma=136,23$ \\
${\text { Cenário } \|^{2}}^{2}$ & $\mu=1.227,61$ & $\sigma=49,76$ & $\mu=2.351,93$ & $\sigma=95,33$ \\
\hline
\end{tabular}

'coleta de dados amostrais de obstrução em arco com alto risco $(n=16)$; ${ }^{2}$ coleta de dados amostrais de obstrução em arco com baixo risco $(n=16)$; ${ }^{3}$ desvio-padrão. Fonte: Goes et al. (2017). 


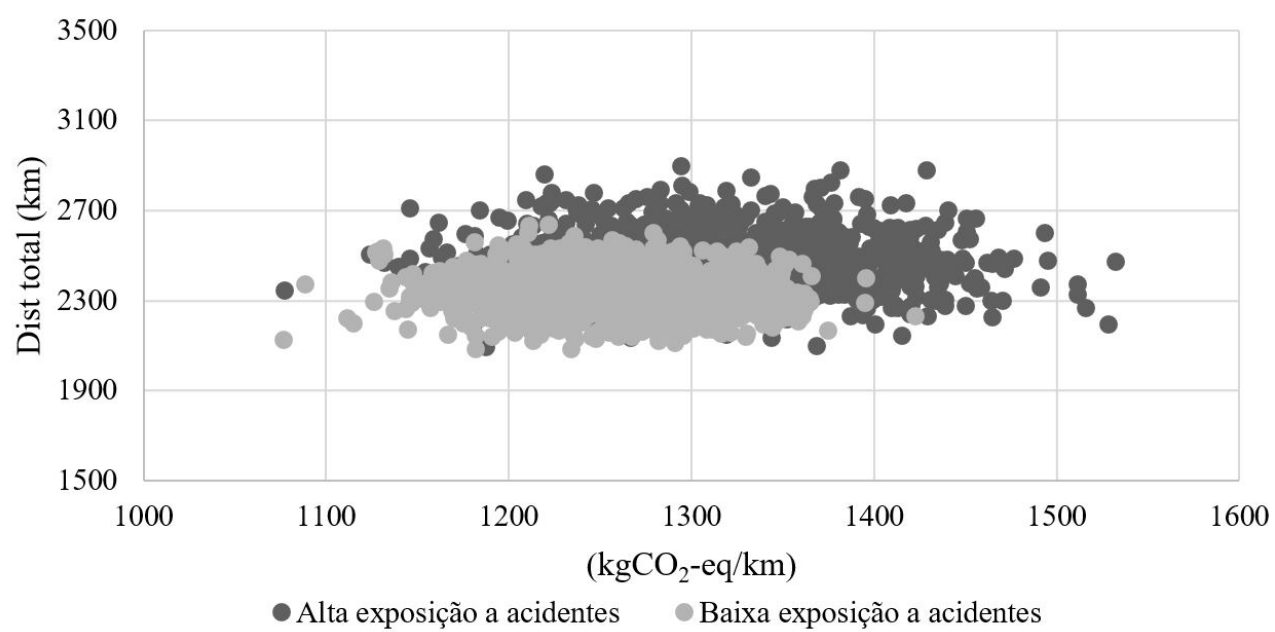

Figura 3 - Relaç̦ão entre distância e emissões por cenário Fonte: Elaborada pelos autores.

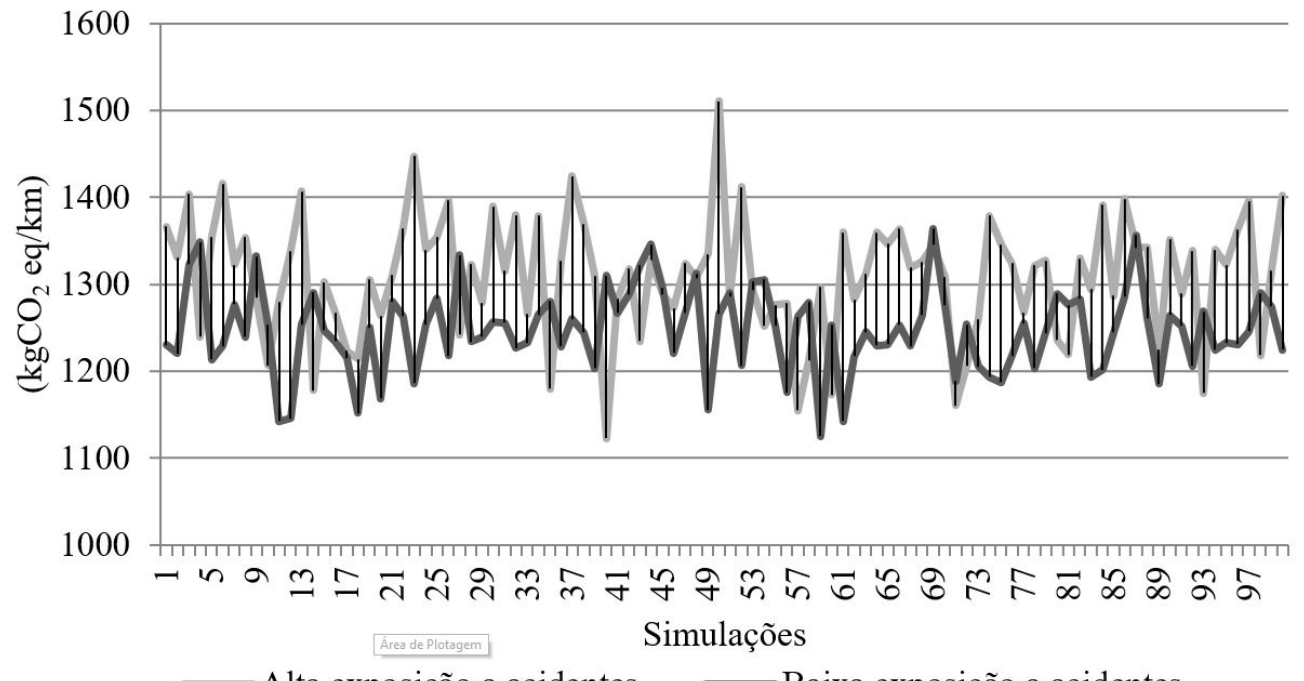

Figura 4 - Simulação das emissões de $\mathrm{CO}_{2}$-eq ao longo do tempo Fonte: Elaborada pelos autores.

A Tabela 5 ilustra a variação dos índices para ambos os cenários. No primeiro caso, a variação do $\mathrm{CO}_{2}$-eq é 9,19\% maior em relação ao status de rede íntegra e apresenta $8,51 \%$ a mais de quilometragem percorrida pelos VUC. No segundo caso, a variação após a simulação foi de 5,54\% ( $\mathrm{CO}_{2}$-eq) e 2,38\% (distância total), respectivamente.

A maior variação dos índices para o cenário I pode ser explicada pelas variáveis significativas de volume médio diário de veículos de carga (Vol_Veic) e velocidade média no trecho (Vel_Méd) adotadas no MPA, visto que, nesse MPA, a variável do volume médio diário do arco possui maior peso no valor total da variável resposta (Cardoso \& Goldner, 2007). No experimento, os arcos apresentados com alto risco de acidente se localizam próximos de polos geradores de tráfego, os quais, ao serem interrompidos, acarretam uma maior quantidade de usuários afetados, portanto aumentos substanciais da distância total percorrida pelos caminhões na rede viária.

No quesito de emissões globais de GEE (Figura 4), a simulação mostra que, em média, os valores de 
Tabela 5 - Resultados da simulação de Monte Carlo

\begin{tabular}{|c|c|c|c|c|}
\hline Cenários & Emissão CO2-eq (kg) & Variação $(\%)^{3}$ & Distância percorrida (km) & Variação (\%) \\
\hline Rede íntegra & $1.192,81$ & - & $2.285,25$ & - \\
\hline Cenário I' & $\mu=1.302,48$ & 9,19 & $\mu=2.479,70$ & 8,51 \\
\hline Cenário || ${ }^{2}$ & $\mu=1.258,83$ & 5,54 & $\mu=2.339,66$ & 2,38 \\
\hline
\end{tabular}

'alto risco de acidente; ${ }^{2}$ baixo risco de acidente; ${ }^{3}$ variação em relação ao cenário de rede íntegra

Fonte: Elaborada pelos autores.

emissões são maiores no caso de ruptura em um arco com alto risco, uma vez que a distância total das viagens aumenta na mesma proporção. Pelos resultados da aplicação, é possível inferir que o atributo de risco está diretamente relacionado a arcos mais vulneráveis para a rede viária. Desse modo, as evidências geradas podem contribuir para análises de vulnerabilidade da rede viária em centros adensados no intuito de reduzir a quantidade de iterações em simulações, além de auxiliar em políticas mitigatórias de emissões de GEE.

\section{Conclusão}

Este artigo buscou verificar a eficácia de um atributo de risco de acidente como direcionador para a análise de vulnerabilidade da rede viária, em termos de aumento da distância e de emissões de $\mathrm{CO}_{2}$-eq, aplicado ao transporte urbano de carga. Entretanto, para compreender de que forma o risco é tratado na literatura, sob forma do fenômeno de interrupção, bem como os aspectos gerais para análises de vulnerabilidade, lançou-se mão de uma RBS como forma de identificar na literatura quais fenômenos de interrupção são utilizados em análises de vulnerabilidade, o cenário (urbano ou regional), as métricas, os métodos e os aspectos ambientais (mitigação de emissões de GEE). Buscou-se, portanto, levantar uma lacuna na literatura ao relacionar vulnerabilidade da rede viária com o risco e os aspectos ambientais, tendo ênfase no transporte urbano de carga.

A RBS evidenciou lacunas quanto a análises que utilizam grupos predefinidos de arcos (para rupturas) em meio urbano, considerando simulação de acidentes como fenômeno de ruptura. Nenhum artigo abordou a emissão de GEE resultante das alterações do fluxo do menor caminho para caminhos alternativos (possivelmente mais longos), levando em conta qualquer tipo de veículo. Tais conclusões preliminares atestam o fator inovador deste trabalho. Ademais, por meio dos resultados das simulações dos cenários de corte, advoga-se que o conceito de risco pode ser empregado como direcionador da vulnerabilidade de uma rede. Devido ao estado de equilíbrio da rede, as alterações de rotas dos motoristas afetados pelo corte de um arco propiciam aumento da distância das viagens de forma agregada, ou seja, envolvendo os demais motoristas que trafegam no mesmo intervalo de tempo.

No experimento, observou-se que os arcos de alto risco de acidente apresentaram aumento de 8,51\% da distância total de viagem quando obstruídos. Em outra vertente, as rupturas realizadas nos arcos de baixo risco apresentaram 2,38\% de acréscimo na distância das viagens da rede, ou seja, trata-se de elos com menor representatividade em caso de ruptura. $\mathrm{O}$ comportamento das emissões de $\mathrm{CO}_{2}$-eq seguiu a mesma tendência da variação da distância total. Em arcos com maior risco, a ruptura ocasiona em aumento médio de $9,19 \%$ da emissão total de $\mathrm{CO}_{2}$-eq, enquanto as rupturas nos arcos com menor risco ocasionaram apenas 5,54\% de aumento nas emissões totais. Apesar da existência de trabalhos que relacionam atributos de fluxo e capacidade (por exemplo, volume de tráfego), o MPA, em trechos inseridos no procedimento de análise de vulnerabilidade, permite a inserção de variáveis significativas que englobam outros fatores de exposição (qualidade do pavimento, presença de motocicletas, interseções semaforizadas etc.), ampliando o escopo de risco. Ademais, relacionar variações no fluxo de veículos, perante interrupções de arcos, com emissões de GEE permite a visualização da problemática de mitigação de emissões sob outra ótica, além do melhoramento da eficiência energética e dos fatores de emissão dos veículos. 
Dessa forma, as conclusões vêm a contribuir com o trabalho de Knoop et al. (2012) no que se refere à triagem dos arcos a ter a vulnerabilidade analisada. Apesar de o tamanho da rede viária modelada ser restrito, a quantidade de cenários modelados para coleta de parâmetros e a simulação de Monte Carlo permitiram avaliar os impactos sob várias fontes de incertezas. Como trabalhos futuros, sugere-se a adoção de novas variáveis significativas do MPA que não tratem apenas do fluxo de tráfego, mas de variáveis, como tipo de frota circulante e condições do pavimento. Tal adoção poderia trazer maior representatividade ao MPA e ao risco de acidentes por si.

\section{Referências}

Aarts, L., \& Van Schagen, I. (2006). Driving speed and the risk of road crashes: a review. Accident; Analysis and Prevention, 38(2), 215-224. http://dx.doi.org/10.1016/j. aap.2005.07.004. PMid:16256932.

Altiparmak, F., Gen, M., Lin, L., \& Paksoy, T. (2006). A genetic algorithm approach for multi-objective optimization of supply chain networks Computers \&. Industrial Engineering (American Institute of Industrial Engineers), 51, 196-215.

Ambituuni, A., Amezaga, J. M., \& Werner, D. (2015). Risk assessment of petroleum product transportation by road: a framework for regulatory improvement. Safety Science, 79, 324-335. http://dx.doi.org/10.1016/j.ssci.2015.06.022.

Asadabadi, A., \& Miller-Hooks, E. (2017). Assessing strategies for protecting transportation infrastructure from an uncertain climate future. Transportation Research Part A, Policy and Practice, 105, 27-41. http://dx.doi. org/10.1016/j.tra.2017.08.010.

Balijepalli, C., \& Oppong, O. (2014). Measuring vulnerability of road network considering the extent of serviceability of critical road links in urban areas. Journal of Transport Geography, 39, 145-155. http://dx.doi.org/10.1016/j. jtrangeo.2014.06.025.

Bevrani, B., Burdett, R. L., Bhaskar, A., \& Yarlagadda, P. K. (2017). A capacity assessment approach for multi-modal transportation systems. European Journal of Operational Research, 263(3), 864-878. http://dx.doi.org/10.1016/j. ejor.2017.05.007.
Brasil. Ministério do Meio Ambiente - MMA. (2013). Inventário Nacional de Emissões Atmosféricas por Veículos Rodoviários. Brasília: Ministério do Meio Ambiente.

Cardoso, G., \& Goldner, L. G. (2007). Desenvolvimento e aplicação de modelos para previsão de acidentes de trânsito. Transportes, 15(2), 43-51.

Chen, A., Yang, C., Kongsomsaksakul, S., \& Lee, M. (2007). Network-based acessibility measures for vulnerability analysis of degradable transportation networks. Networks and Spatial Economics, 7(3), 241-256. http://dx.doi. org/10.1007/s11067-006-9012-5.

Cheshmehgaz, H. R., Ishak Desa, M., \& Wibowo, A. (2013). An effective model of multiple multi-objective evolutionary algorithms with the assistance of regional multi-objective evolutionary algorithms: VIPMOEAs. Applied Soft Computing, 13(5), 2863-2895. http://dx.doi. org/10.1016/j.asoc.2012.04.027.

Colella, D. A. T., Lima, E. P., \& Demarchi, S. H. (2004) Calibração e validação do modelo fluxo-velocidade do Integration para vias urbanas semaforizadas. In Anais do XVIII Congresso de Pesquisa e Ensino em Transportes (Vol. 1, pp. 453-564). Rio de Janeiro: ANPET.

Conca, A., Ridella, C., \& Sapori, E. (2016). A risk assessment for road transportation of dangerous goods: a routing solution. Transportation Research Procedia, 14, 2890-2899. http://dx.doi.org/10.1016/j.trpro.2016.05.407.

Cunha, M. S., Fo. (2014). Avaliação da vulnerabilidade da rede de transporte rodoviário de carga no Brasil (Dissertação de mestrado). Departamento de Engenharia Civil e Ambiental, Universidade de Brasília, Brasilia.

Demirel, H., Kompil, M., \& Nemry, F. (2015). A framework to analyze the vulnerability of European road networks due to Sea-Level Rise (SLR) and sea storm surges. Transportation Research Part A, Policy and Practice, 81, 62-76. http:// dx.doi.org/10.1016/j.tra.2015.05.002.

Faturechi, R., \& Miller-Hooks, E. (2014). Measuring the performance of transportation infrastructure systems in disasters: a comprehensive review. Journal of Infrastructure Systems, 21(1), 1-15. http://dx.doi.org/10.1061/(ASCE) IS.1943-555X.0000212.

Fialkoff, M. R., Omitaomu, O. A., Peterson, S. K., \& Tuttle, M. A. (2017). Using geographic information science to evaluate legal restrictions on freight transportation routing in disruptive scenarios. International Journal of 
Critical Infrastructure Protection, 17, 60-74. http://dx.doi. org/10.1016/j.ijcip.2016.12.001.

Gen, M., Altiparmak, F., \& Lin, L. (2006). A genetic algorithm for two stage transportation problem using priority-based encoding. OR-Spektrum, 28(3), 337-354. http://dx.doi. org/10.1007/s00291-005-0029-9.

Goes, G. V., \& Bertoncini, B. V. (2016). Modelo de estimação de custos do transporte urbano de cargas com base na vulnerabilidade da rede viária. Journal of Transport Literature, 10(2), 30-34. http://dx.doi.org/10.1590/22381031.jtl.v10n2a6.

Goes, G. V., Bertoncini, B. V., \& Dagosto, M. A. (2017). Risco de acidente como conceito direcionador para análise de vulnerabilidade da rede viária. In Anais do XXXI Congresso Nacional de Pesquisa e Ensino em Transporte da ANPET (pp. 144-156). Rio de Janeiro: ANPET.

Greibe, P. (2003). Accident prediction models for urban roads. Accident, Analysis and Prevention, 35(2), 273-285. http://dx.doi.org/10.1016/S0001-4575(02)00005-2. PMid:12504148.

Haimes, Y. Y. (1998). Risk modeling, assessment, and management. New York: Wiley.

Huang, Z., Kuang, A., Fan, W., \& Zhou, Q. (2012). Impact of traveler information on road network travel time reliability. Journal of Transportation Systems Engineering and Information Technology, 12(6), 93-99. http://dx.doi. org/10.1016/S1570-6672(11)60235-3.

Inanloo, B., Tansel, B., Shams, K., Jin, X., \& Gan, A. (2016). A decision aid GIS-based risk assessment and vulnerability analysis approach for transportation and pipeline networks. Safety Science, 84, 57-66. http://dx.doi.org/10.1016/j. ssci.2015.11.018.

Jenelius, E., \& Mattsson, L. G. (2012). Road network vulnerability analysis of area-covering disruptions: A gridbased approach with case study. Transportation Research Part A, Policy and Practice, 46(5), 746-760. http://dx.doi. org/10.1016/j.tra.2012.02.003.

Jenelius, E., Petersen, T., \& Mattsson, L. G. (2006). Importance and exposure in road network vulnerability analysis. Transportation Research Part A, Policy and Practice, 40(7), 537-560. http://dx.doi.org/10.1016/j.tra.2005.11.003.

Kermanshah, A., \& Derrible, S. (2016). A geographical and multi-criteria vulnerability assessment of transportation networks against extreme earthquakes. Reliability Engineering
\& System Safety, 153, 39-49. http://dx.doi.org/10.1016/j. ress.2016.04.007.

Knoop, V. L., Hoogendoorn, S. P., \& van Zuylen, H. J. (2007). Approach to critical link analysis of robustness for dynamical road networks. In A. Schadschneider, T. Pöschel, R. Kühne, M. Schreckenberg, \& D. E. Wolf (Eds.), Traffic and granular flow'05 (pp. 393-402). Berlin: Springer Berlin Heidelberg. http://dx.doi.org/10.1007/978-3-540-47641-2_35.

Knoop, V. L., Snelder, M., van Zuylen, H. J., \& Hoogendoorn, S. P. (2012). Link-level vulnerability indicators for realworld networks. Transportation Research Part A, Policy and Practice, 46(5), 843-854. http://dx.doi.org/10.1016/j. tra.2012.02.004.

Leng, J., Zhai, J., Li, Q., \& Zhao, L. (2018). Construction of road network vulnerability evaluation index based on general travel cost. Physica A, 493, 421-429. http://dx.doi. org/10.1016/j.physa.2017.11.018.

Lord, D., Manar, A., \& Vizioli, A. (2005). Modeling crashflow-density and crash-flow-V/C ratio relationships for rural and urban freeway segments. Accident; Analysis and Prevention, 37(1), 185-199. http://dx.doi.org/10.1016/j. aap.2004.07.003. PMid:15607290.

Luathep, P., Sumalee, A., Ho, H. W., \& Kurauchi, F. (2011). Large-scale road network vulnerability analysis: a sensitivity analysis based approach. Transportation, 38(5), 799-817. http://dx.doi.org/10.1007/s11116-011-9350-0.

Lyons, G., (2016). Getting smart about urban mobility - aligning the paradigms of smart and sustainable. Transportation Research Part A: Policy and Practice (In press). https://doi.org/10.1016/j.tra.2016.12.001.

Mattsson, L. G., \& Jenelius, E. (2015). Vulnerability and resilience of transport systems - a discussion of recent research. Transportation Research Part A, Policy and Practice, 81, 16-34. http://dx.doi.org/10.1016/j.tra.2015.06.002.

Muriel-Villegas, J. E., Alvarez-Uribe, K. C., Patiño-Rodríguez, C. E., \& Villegas, J. G. (2016). Analysis of transportation networks subject to natural hazards - Insights from a Colombian case. Reliability Engineering \& System Safety, 152,151-165. http://dx.doi.org/10.1016/j.ress.2016.03.006.

Myhre, G., Shindell, D., Bréon, F.-M., Collins, W., Fuglestvedt, J., Huang, J., Koch, D., Lamarque, J.-F., Lee, D., Mendoza, B., Nakajima, T., Robock, A., Stephens, G., Takemura, T., \& Zhang, H. (2013). Anthropogenic and natural radiative forcing. In T. F. Stocker, D. Qin, G.-K. Plattner, M. Tignor, S. K. Allen, J. Boschung, A. Nauels, Y. Xia, V. Bex \&, P. M. Midgley 
(Eds.), Climate Change 2013: The Physical Science Basis. Contribution of Working Group I to the Fifth Assessment Report of the Intergovernmental Panel on Climate Change (pp. 659-740). Cambridge: University Press. http://doi. org/10.1017/ CB09781107415324.018.

Ngai, E. W. T., \& Wat, F. K. T. (2002). A literature review and classification of electronic commerce research. Information \& Management, 39(5), 415-429. http://dx.doi.org/10.1016/ S0378-7206(01)00107-0.

Nord, J. H., \& Nord, G. D. (1995). Mis research: Journal status assessment and analysis. Information \& Management, 29(1), 29-42. http://dx.doi.org/10.1016/0378-7206(95)00010-T.

Oliveira, E. L., \& Portugal, L. S., \& Porto, W., Jr. (2016). Indicators of reliability and vulnerability: Similarities and differences in ranking links of a complex road system. Transportation Research Part A, Policy and Practice, 88, 195-208. http://dx.doi.org/10.1016/j.tra.2016.04.004.

Oliveira, M. J. (2001). Dinâmica estocástica e irreversibilidade (Vol. 35). São Paulo: EdUSP.

Scott, D. M., Novak, D. C., Aultman-Hall, L., \& Guo, F. (2006). Network Robustness Index: a new method for identifying critical links and evaluating the performance of transportation networks. Journal of Transport Geography, 14(3), 215-227. http://dx.doi.org/10.1016/j.jtrangeo.2005.10.003.

Sullivan, J. L., Novak, D. C., Aultman-Hall, L., \& Scott, D. M. (2010). Identifying critical road segments and measuring system-wide robustness in transportation networks with isolating links: a link-based capacity-reduction approach. Transportation Research Part A, Policy and Practice, 44(5), 323-336. http://dx.doi.org/10.1016/j.tra.2010.02.003.

Tampère, C. M. J., Stada, J., Immers, B., Peetermans, E., \& Organe, K. (2008). Methodology for identifying vulnerable sections in a national road network. Transportation Research Record: Journal of the Transportation Research Board, (1), 1-10. http://dx.doi.org/10.3141/2012-01.

Taylor, M. A. P., Sekhar, S. V. C., \& D’Este, G. M. (2006). Application of accessibility based methods for vulnerability analysis of strategic road networks. Networks and Spatial Economics, 6(3-4), 267-291. http://dx.doi.org/10.1007/ s11067-006-9284-9.

Thomé, A. M. T., Scavarda, L. F., \& Scavarda, A. J. (2016). Conducting systematic literature review in operations management. Production Planning and Control, 27(5), 408420. http://dx.doi.org/10.1080/09537287.2015.1129464.

Thorisson, H., \& Lambert, J. H. (2017). Multiscale identification of emergent and future conditions along corridors of transportation networks. Reliability Engineering \& System Safety, 167, 255-263. http://dx.doi.org/10.1016/j. ress.2017.06.005.

Transportation Research Board - TRB. (1998). Travel estimation techniques for urban planning (NCHR Report, No. 365). Washington: Transportation Research Board.

Wardrop, J. G. (1952). Road paper. Some theoretical aspects of road traffic research. Proceedings - Institution of Civil Engineers, 1(3), 325-362. http://dx.doi.org/10.1680/ ipeds.1952.11259.

Yang, H., Lo, H. K., \& Tang, W. H. (2000). Travel time versus capacity reliability of a road network. In M. G. H. Bell, \& C. Cassir (Eds.), Reliability of transport networks (pp. 119138). Baldock, Herts: Research Studies Press.

Recebido: Ago. 30, 2017

Aprovado: Fev. 15, 2018 